\title{
Prediction of functional outcome in patients with convulsive status epilepticus: the END-IT score
}

Qiong Gao, Tang-peng Ou-Yang, Xiao-long Sun, Feng Yang, Chen Wu, Tao Kang, Xiao-gang Kang and Wen Jiang*

\begin{abstract}
Background: Prediction of the functional outcome for patients with convulsive status epilepticus (CSE) has been a challenge. The aim of this study was to characterize the prognostic factors and functional outcomes of patients after CSE in order to develop a practicable scoring system for outcome prediction.
\end{abstract}

Methods: We performed a retrospective explorative analysis on consecutive patients diagnosed with CSE between March, 2008 and November, 2014 in a tertiary academic medical center in northwest China. The modified Rankin Scale (mRS) was used to measure the functional outcome at three months post discharge.

Results: A total of 132 CSE patients was included, with a median age of 25.5 years and $60.6 \%$ were male. Three months post discharge, an unfavorable outcome with mRS of 3-6 was seen in 62 (47.0\%) patients, 25 (18.9\%) of whom died. Logistic regression analysis revealed that encephalitis $(p=0.029)$, nonconvulsive SE $(p=0.018)$, diazepam resistance $(p=0.005$ ), image abnormalities (unilateral lesions, $p=0.027$; bilateral lesions or diffuse cerebral edema, $p<0.001)$ and tracheal intubation $(p=0.032)$ were significant independent predictors for unfavorable outcomes. Based on the coefficients in the model, these predictors were assigned a value of 1 point each, with the exception of the image, creating a 6-point scoring system, which we refer to as END-IT, for the outcome prediction of CSE. The area under the receiver operating characteristic curve for the END-IT score was 0.833 and using a cutoff point of 3 produced the highest sum sensitivity ( $83.9 \%$ ) and specificity (68.6\%). Compared with status epilepticus severity score (STESS) and Epidemiology-based Mortality score in SE (EMSE), END-IT score showed better discriminative power and predictive accuracy for the outcome prediction.

Conclusions: We developed an END-IT score with a strong discriminative power for predicting the functional outcome of CSE patients. External prospective validation in different cohorts is needed for END-IT score.

\section{Background}

Convulsive status epilepticus (CSE) is a common, lifethreatening neurological disorder [1]. Even with prompt treatment, the mortality rate remains high, ranging from 7.6 to $39 \%$ [2], and more than $10 \%$ of survivors develop neurological and cognitive disabilities [3, 4]. An accurate quantification of CSE severity and a reliable predictor of functional outcomes would be beneficial for clinicians in optimizing individualized patient management and communicating with relatives and other healthcare professionals.

\footnotetext{
* Correspondence: jiangwen@fmmu.edu.cn

Department of Neurology, Xijing Hospital, Fourth Military Medical University, Xi'an 710032, PR China
}

(C) 2016 Gao et al. Open Access This article is distributed under the terms of the Creative Commons Attribution 4.0 International License (http://creativecommons.org/licenses/by/4.0/), which permits unrestricted use, distribution, and reproduction in any medium, provided you give appropriate credit to the original author(s) and the source, provide a link to the Creative Commons license, and indicate if changes were made. The Creative Commons Public Domain Dedication waiver (http://creativecommons.org/publicdomain/zero/1.0/) applies to the data made available in this article, unless otherwise stated.
Currently, two scores for SE outcome prediction are available, the Status Epilepticus Severity Score (STESS) $[5,6]$ and the Epidemiology based Mortality score in SE (EMSE) [7]. The STESS was developed from a retrospective study and was based on four variables at the time of presentation: history of seizures, age, seizure type, and consciousness impairment [6]. The EMSE score was derived from a retrospective, exploratory analysis based on epidemiological data and taking into consideration etiology, age, comorbidity and electroencephalogram (EEG) data [7]. Both of these scores have been primarily used to predict survival vs. death in the hospital setting. However, no scoring system currently exists for the purpose of predicting the functional outcome of patients with CSE once they have been discharged. 
Here we performed a retrospective cohort study, on the basis of a prospective registry for SE, with two aims: (1) to identify independent prognostic factors associated with the functional outcome of patients three months after discharge from the hospital setting by analyzing demographic data, clinical features, neuroimages, and the treatment of the disease in the hospital; and (2) to establish a prognostic score by incorporating these variables according to their determined relative contributions to the resulting functional outcome.

\section{Methods}

\section{Study setting and patients}

This retrospective analysis utilized a registry for SE from the neurological intensive care unit (N-ICU) at Xijing Hospital, a tertiary academic medical care institution with 3200-beds in Xi'an, China. All patients with CSE from March 2008 to November 2014 were recruited for this study as long as they were older than 12 years of age. CSE was defined as 30 minutes or more of (1) continuous motor seizure activity or (2) recurrent seizure activity without regaining full consciousness between episodes [8]. Subjects with CSE from cerebral anoxia were excluded due to the high rate of mortality [9]. All patients were followed up for at least three months after discharge from Xijing Hospital. For this, two to three telephone numbers (both mobile and landline) were collected from the patients' relatives at the time of registry. The present study was approved by the ethics committee of the Xijing Hospital and was carried out in agreement with Chinese laws and the Helsinki declaration relative to patients' rights.

\section{SE treatment procedure}

All of the CSE patients were treated according to the established hospital protocol, which was based upon published guideline recommendations $[10,11]$. Intravenous (IV) diazepam was administered as a first-line antiepileptic drug (AED), followed by IV second-line AEDs if SE persisted (e.g., valproic acid, phenobarbital). If the first- and second-line AEDs failed to treat the patient, third-line treatments with IV anesthetics (e.g., midazolam, propofol) were initiated. Ketamine, isoflurane, and a ketogenic diet were used as a fourth-line therapy.

After admission, each patient received continuous bedside video-EEG monitoring (Solar $2000 \mathrm{~N}$, Solar Electronic Technologies Co., Ltd, Beijing, China) for guiding the AED treatment and detecting the occurrence of nonconvulsive SE (NCSE). NCSE was defined as more than 30 minutes of continuous seizure activity seen on an EEG, with or without subtle motor movements $[10,12]$. Upon discharge from the Xijing Hospital, patients were transferred to secondary hospitals where they received rehabilitation treatment.

\section{Definition of the predictor variables}

The following predictor variables with a possible association with the recovery of CSE were chosen for statistical analysis. Three of the chosen variables concerned the demographic and medical history: (1) age; (2) gender; and (3) history of epilepsy, coded as being present or absent.

Two variables were extracted from the clinical examination on N-ICU admission: (1) Glasgow Coma Scale (GCS), dichotomized as a score ranging from 3-8 or ranging from 9-15; and (2) pupillary light reflex, coded as sensitive on both sides, or slow or absence on one side or two sides.

One variable concerned the SE etiology. Given that encephalitis constitutes most of the SE cases, presenting as an acute symptomatic etiology in developing countries, and is related to poor outcome [2,13-17], the SE etiology was categorized as either encephalitis or non-encephalitis.

Four variables were extracted from the SE treatment: (1) resistance to diazepam, coded as Yes or No; (2) SE duration (time to seizure control), dichotomized as $<2$ hrs or $\geq 2 \mathrm{hrs}$, which is based on previous studies showing that CSE persisting more than 2 hrs was often defined as refractory SE and the patients with CSE exceeding 2 hrs were more likely to have a poor outcome than those with less than 2 hrs [18-20]; (3) drug induced coma; and (4) use of three or more types of intravenous AEDs, both coded as Yes or No.

Three variables concerned comorbidity: (1) complicated with NCSE; (2) tracheal intubation, both coded as Yes or No; and (3) the Charlson's Comorbidity Index (CCI), classified into three categories: 0 point, 1 to 2 points, 3 points or more $[7,21]$.

Brain images were also considered as a predictor variable. According to the distribution of responsible lesions on images for SE, we classified brain images into three different categories: no responsible lesion, unilateral responsible lesions, and bilateral responsible lesions or diffuse cerebral edema. We reviewed the first and subsequent study findings of computed tomography (CT) and magnetic resonance imaging (MRI) scans after SE onset and only recorded the most serious findings. The classification of the image was determined by two experienced neurologists unaware of the final outcome, who based their decisions on both pictures and the official radiology reports, and a consensus was reached.

\section{Definition of outcomes}

The clinical outcome was independently assessed three months after discharge from the Xijing Hospital by a trained neurologist, who was blinded to the clinical data, via a telephone interview. The mRS was used to measure the disability of the patients [22]; this scale comprises seven different levels of outcomes ranging from 0 (no symptoms) to 5 (severe disability) and 6 (death). In cases 
where the patient was unable to complete the interview, the mRS score was determined by interviewing the patient's caregiver. For the purpose of statistical analysis, we defined the score range of $0-2$ (independence) of $\mathrm{mRS}$ as a favorable outcome, and the score range of 3-6 (death or dependence with regard to activities of daily living) as an unfavorable outcome.

\section{Statistical analysis}

Continuous variables were expressed as mean \pm standard deviation (normally distributed), as medians and interquartile ranges (IQR, not normally distributed), or, if they were categorical variables, they were expressed as counts and percentages. We first conducted univariate comparisons for each outcome using the $\chi^{2}$ test for the categorical variables and the Mann-Whitney $U$ test or Student's $t$-test for the continuous variables. A backward stepwise multivariate logistic regression was then performed in order to identify independent outcome predictors among those found to have a $p<0.05$ on the univariate analysis. Points were then assigned to each independent risk factor by dividing its $\beta$-coefficient in the model by the lowest $\beta$-coefficient and rounding to the nearest integer. Thus, a predictive score was created for each subject by adding the points determined for each factor, with higher scores corresponding to a higher likelihood of an unfavorable outcome for CSE patients. The performance of the predictive score was then evaluated by calculating the area under the receiver operating characteristic curve (ROC). Using the ROC curve, the best cutoff value of the score that was able to predict the primary end point was determined. Predictive accuracy was calculated as the average of sensitivity and specificity. Statistical analyses were performed using the SPSS 18.0 and Matlab 2012a. All tests were two-sided, and a $\mathrm{p}$ value of less than 0.05 was considered statistically significant in univariate and multivariate analyses, as was a $p$ value lower than 0.0083 for comparison tests on performances [23].

\section{Results}

The patient flow chart is shown in Fig. 1. A total of 132 CSE patients were included in the study, each of whom was able to be followed up. The median age of the sample was 25.5 (IQR, 17-48) years and $60.6 \%$ of them were men. The most common cause of CSE in our sample was encephalitis, accounting for $35.6 \%$, followed by AED low levels/withdrawal (13.6\%) and cerebrovascular diseases (11.4\%), as shown in Table 1. At three months post discharge, $70(53.0 \%)$ patients presented with a

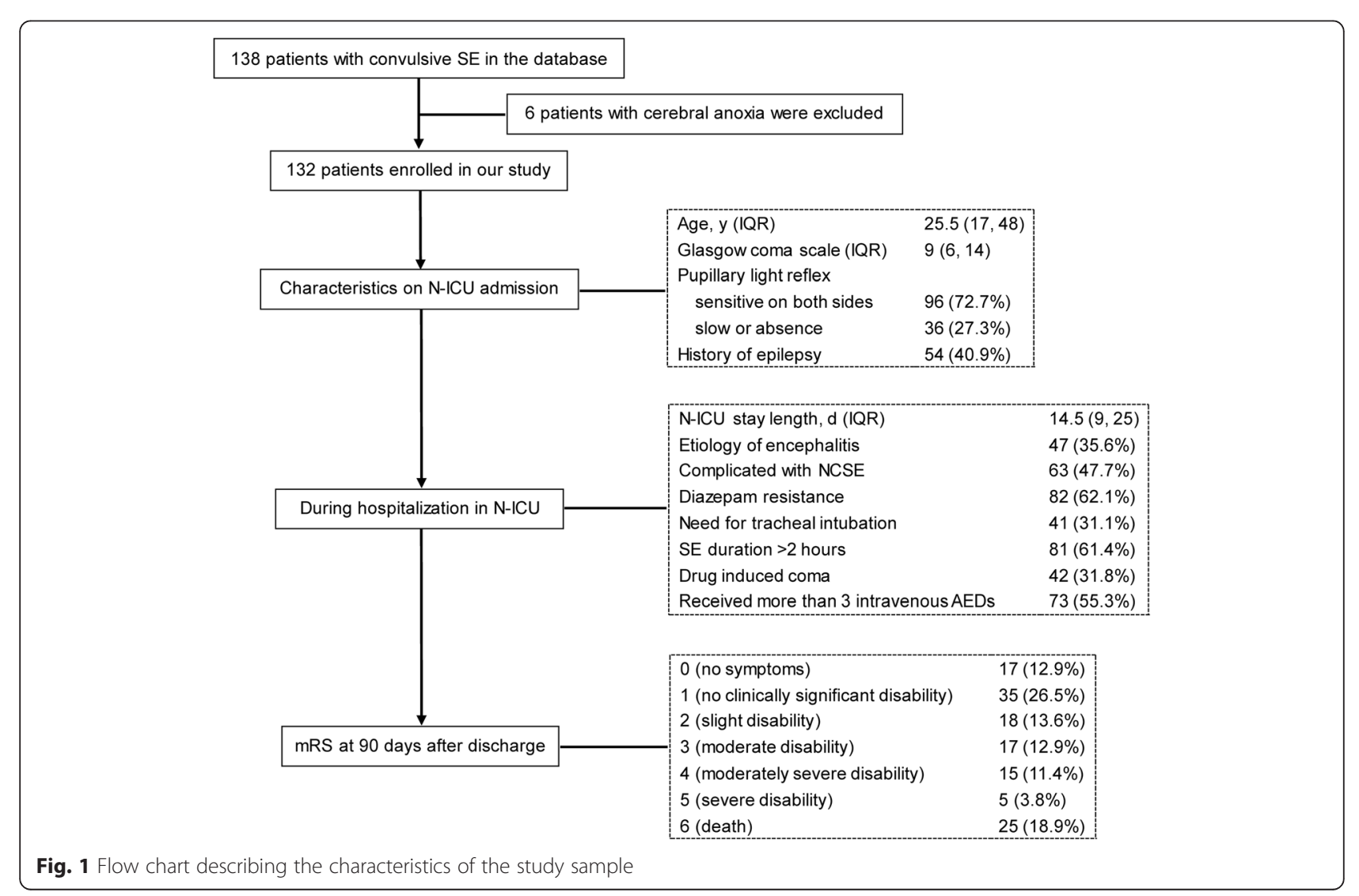


Table 1 Causes of convulsive status epilepticus

\begin{tabular}{|c|c|c|c|}
\hline Etiology & Total No. (\%) & $\begin{array}{l}\text { mRS (0-2) } \\
\text { No. }(\%)\end{array}$ & $\begin{array}{l}\text { mRS (3-6) } \\
\text { No. (\%) }\end{array}$ \\
\hline \multicolumn{4}{|l|}{ Broad categories } \\
\hline Acute symptomatic & $81(61.4)$ & $36(51.4)$ & $45(72.6)$ \\
\hline Remote symptomatic & $26(19.7)$ & $15(21.4)$ & $11(17.7)$ \\
\hline Progressive symptomatic & $5(3.8)$ & $4(5.7)$ & $1(1.6)$ \\
\hline Unknown & $20(15.2)$ & $15(21.4)$ & $5(8.1)$ \\
\hline \multicolumn{4}{|l|}{ Individual categories } \\
\hline Encephalitis & 47 (35.6) & $15(21.4)$ & $32(51.6)$ \\
\hline Viral & $37(28.0)$ & $13(18.6)$ & $24(38.7)$ \\
\hline Tuberculous & $4(3.0)$ & $2(2.9)$ & $2(3.2)$ \\
\hline Autoimmune & $4(3.0)$ & 0 & $4(6.5)$ \\
\hline Bacterial & $1(0.8)$ & 0 & $1(1.6)$ \\
\hline Cryptococcal & $1(0.8)$ & 0 & $1(1.6)$ \\
\hline AED low levels/withdrawal & $18(13.6)$ & $6(8.6)$ & $12(19.4)$ \\
\hline Acute cerebrovascular diseases & $15(11.4)$ & $7(10.0)$ & $8(12.9)$ \\
\hline Metabolism/alcoholism & $11(8.3)$ & $10(14.3)$ & $1(1.6)$ \\
\hline Acute trauma & $2(1.5)$ & $2(2.9)$ & 0 \\
\hline Remote trauma & $12(9.1)$ & $9(12.9)$ & $3(4.8)$ \\
\hline Tumor & $3(2.3)$ & $2(2.9)$ & $1(1.6)$ \\
\hline Systematic infection & $2(1.5)$ & $2(2.9)$ & 0 \\
\hline Mitochondrial encephalopathy & $1(0.8)$ & $1(1.4)$ & 0 \\
\hline Cerebral lupus & $1(0.8)$ & $1(1.4)$ & 0 \\
\hline Cryptogenic & $12(9.1)$ & $10(14.3)$ & $2(3.2)$ \\
\hline Two or more causes & $8(6.1)$ & $5(7.1)$ & $3(4.8)$ \\
\hline
\end{tabular}

$m R S$ modified Rankin Scale, $A E D$ antiepileptic drug

favorable outcome (mRS 0-2), while $62(47.0 \%)$ patients had an unfavorable outcome (mRS 3-6), among whom $25(18.9 \%)$ died.

The demographic, clinical, and neuroimage features of the patients during hospitalization in the N-ICU are summarized in Table 2 and Additional file 1. Univariate analysis indicated that age $(p=0.075)$, gender $(p=0.387)$, history of epilepsy $(p=0.821)$, pupillary light reflex on admission $(p=0.109)$, and CCI $(p=0.260)$ did not correlate significantly with the outcome noted at three months post discharge (Table 2). An unfavorable outcome was more likely if the patient had initially presented with a lower GCS score during the time of admission, experienced diazepam resistance and had a longer SE duration, suffered from encephalitis and tracheal intubation, received more than three intravenous AEDs and had a drug induced coma, progressed to NCSE, and displayed abnormal brain images $(p<0.05)$. These variables were then entered into the multivariate logistic regression model, and the results indicated that only encephalitis, NCSE, diazepam resistance, imaging abnormalities, and tracheal intubation were significant independent predictors for an unfavorable outcome (Table 3).

In order to establish a straightforward prognostic indicator to be used in clinical practice, we developed a scoring system comprised of the aforementioned five risk factors and named it END-IT, which is an acronym for encephalitis, NCSE, diazepam resistance, image abnormalities and tracheal intubation. Each of the five identified factors was then assigned an integer score based on the magnitude of their determined contribution to adverse events in the multivariate model. The detailed allocation of the scoring points is listed in Table 4 . Each of the variables was assigned one point, with the exception of the image, in which unilateral lesions were given one point and bilateral lesions or the presence of diffuse cerebral edema were given two points. The outcome of CSE can be estimated for an individual patient by summing the points of each predictor resulting in a total point score ranging from 0 to 6 . The probability of unfavorable outcome increases as the score increases (Fig. 2).

The ROC curve for the weighted score was shown to have good discriminative power with an area under the curve (AUC) of 0.833 (95 \% CI, 0.758-0.892) (Fig. 3). The validity of the END-IT score in assessing the threemonth post discharge outcome in this cohort is summarized in Table 5. The cut-off point of 3 of 6 produced the optimal sum of sensitivity and specificity for the prediction of an unfavorable outcome. Fifty-two $(83.9 \%)$ of the 62 patients with an unfavorable outcome had an END-IT score of 3 or greater and $48(68.6 \%)$ of the 70 patients who presented with a favorable outcome had a score of 0 to 2. Using this cutoff value, the END-IT score had a high sensitivity of $83.9 \%$ with a positive predictive value (PPV) of $70.3 \%$ and a specificity of $68.6 \%$ with a negative predictive value (NPV) of $82.8 \%$ for the prediction of functional outcomes (Table 5). The new prognostic score achieved a predictive accuracy of $76.22 \%$.

STESS and EMSE-score using etiology-age-comorbidityEEG (EMSE-EACE) were also calculated in this study cohort and compared with the END-IT score in the predictive power of outcome. Figure 3 showed that the END-IT score $(\mathrm{AUC}=0.833$, accuracy $=76.22 \%$ ) had better discriminative power and predictive accuracy than STESS (AUC $=0.573$, accuracy $=57.98 \%$ ) and EMSE-EACE $(\mathrm{AUC}=0.683$, accuracy $=67.70 \%)$ in the prediction of functional outcomes. The comparisons of sensitivity, specificity, positive (PPV) and negative (NPV) predictive value and number of correctly classified (CC) patients between STESS, EMSE-EACE and END-IT are shown in Fig. 4. END-IT score was significantly superior to STESS with cutoff levels 3 and 4 (STESS-3: 3 or more points indicate bad outcome, i.e., death; STESS-4: 4 or more points indicate bad outcome, i.e., death): NPV of 
Table 2 Univariate analysis

\begin{tabular}{|c|c|c|c|c|}
\hline Variable & Total $(n=132)$ & $m R S: 0-2(n=70)$ & $m R S: 3-6(n=62)$ & $P$ \\
\hline Age, median (IQR), y & $25.5(17.0,48.0)$ & $24(17.0,34.5)$ & $31(18.8,54.3)$ & 0.075 (Mann-Whitney U) \\
\hline Gender, male, No. (\%) & $80(60.6)$ & $40(57.1)$ & $40(64.5)$ & $0.387\left(x^{2}\right)$ \\
\hline History of epilepsy, No. (\%) & $54(40.9)$ & $28(40.0)$ & $26(41.9)$ & $0.821\left(x^{2}\right)$ \\
\hline GCS on admission 3-8, No. (\%) & $54(40.9)$ & $22(31.4)$ & $32(51.6)$ & $0.019\left(x^{2}\right)$ \\
\hline Pupillary light reflex on admission, slow or absence, No. (\%) & $36(27.3)$ & $15(21.4)$ & $21(33.9)$ & $0.109\left(x^{2}\right)$ \\
\hline Encephalitis, No. (\%) & $47(35.6)$ & $15(21.4)$ & $32(51.6)$ & $<0.001\left(x^{2}\right)$ \\
\hline Complicated with NCSE, No. (\%) & $63(47.7)$ & $23(32.9)$ & $40(64.5)$ & $<0.001\left(x^{2}\right)$ \\
\hline Diazepam resistance, No. (\%) & $82(62.1)$ & $32(45.7)$ & $50(80.6)$ & $<0.001\left(x^{2}\right)$ \\
\hline Image & & & & $0.007\left(x^{2}\right)$ \\
\hline unilateral lesions, No. (\%) & $28(21.2)$ & $16(22.9)$ & $12(19.4)$ & \\
\hline bilateral lesions/diffuse cerebral edema, No. (\%) & $63(47.7)$ & $25(35.7)$ & $38(61.3)$ & \\
\hline Tracheal intubation, No. (\%) & $41(31.1)$ & $11(15.7)$ & $30(48.4)$ & $<0.001\left(x^{2}\right)$ \\
\hline Duration >2 h, No. (\%) & $81(61.4)$ & $34(48.6)$ & $47(75.8)$ & $0.001\left(x^{2}\right)$ \\
\hline Drug induced coma, No. (\%) & $42(31.8)$ & $15(21.4)$ & $27(43.5)$ & $0.006\left(x^{2}\right)$ \\
\hline Usage of $\geq 3$ types vAEDs, No. (\%) & $73(55.3)$ & $32(45.7)$ & $41(66.1)$ & $0.019\left(x^{2}\right)$ \\
\hline $\mathrm{CCl}$ & & & & $0.260\left(x^{2}\right)$ \\
\hline $1-2$ & $36(27.3)$ & $17(24.3)$ & 19 (30.6) & \\
\hline 3 or more & $18(13.6)$ & $5(7.1)$ & $13(21.0)$ & \\
\hline
\end{tabular}

mRS modified Rankin Scale, IQR interquartile range, GCS Glasgow coma scale, NCSE nonconvulsive status epilepticus, SE status epilepticus, vAEDs intravenous antiepileptic drugs, $\mathrm{CCl}$ Charlson's Comorbidity Index

END-IT $82.76 \%$, STESS-3 $55.45 \%(\mathrm{p}=0.0000030)$, and STESS-4 $52.80 \%(\mathrm{p}=0.000000099)$; CC of END-IT 75.76 $\%$, STESS-4 $52.27 \%(\mathrm{p}=0.00037)$. Compared with EMSEEACE with cutoff 64, END-IT score showed a better tendency in NPV and CC but there was no statistically significant difference between them.

\section{Discussion}

A straightforward prognostic scoring system was developed in this study in order to determine the functional outcome of patients with CSE. To make full use of the available clinical data and to systematically consider the progression of SE, our score was initially designed to incorporate the demographics, clinical characteristics, and treatment responses, as well as the available neuroimages. The statistical analysis showed that encephalitis,
NCSE, diazepam resistance, image abnormalities and tracheal intubation were significant independent predictors of functional outcome at three months post discharge from the hospital. Using these predictors and their regression coefficients in the model, we developed a simple sixpoint score, termed END-IT, in order to predict the probability of an unfavorable outcome in CSE. The END-IT score was shown to have a good predictive accuracy as well as good discriminative power, thus providing families and clinicians with better information regarding the prognosis. As the function outcome was known during the process of score generation, this study is explorative, i.e., hypothesis generating.

Given that unfavorable outcome at three months after discharge could be caused by many other factors independent of SE, e.g., car accidents, myocardial infarction,

Table 3 Multivariable analysis

\begin{tabular}{|c|c|c|c|c|}
\hline Variable & Coefficient & Odds ratio $(95 \% \mathrm{Cl})$ & $P$ & Weighted integer coefficient \\
\hline Encephalitis & 1.105 & 3.018 (1.119 to 8.141$)$ & 0.029 & 1 \\
\hline NCSE & 1.072 & $2.922(1.201$ to 7.109$)$ & 0.018 & 1 \\
\hline Diazepam resistance & 1.361 & 3.899 (1.519 to 10.007$)$ & 0.005 & 1 \\
\hline \multicolumn{5}{|l|}{ Image } \\
\hline unilateral lesions & 1.479 & 4.386 (1.188 to 16.199$)$ & 0.027 & 1 \\
\hline bilateral lesions/diffuse cerebral edema & 1.979 & 7.236 (2.414 to 21.690$)$ & $<0.001$ & 2 \\
\hline Tracheal intubation & 1.093 & 2.982 (1.099 to 8.092) & 0.032 & 1 \\
\hline
\end{tabular}


Table 4 Point allocation for the END-IT score based on regression coefficients

\begin{tabular}{lll}
\hline Relative factor & Categories & Points \\
\hline Encephalitis & Yes & 1 \\
& No & 0 \\
NCSE & Yes & 1 \\
& No & 0 \\
Diazepam resistance & Yes & 1 \\
& No & 0 \\
Image & bilateral lesions/diffuse cerebral edema & 2 \\
& unilateral lesions & 1 \\
& no responsible lesion & 0 \\
Tracheal intubation & Yes & 1 \\
& No & 0
\end{tabular}

NCSE nonconvulsive status epilepticus

or trauma, it is necessary to exclude these influences in investigating the functional outcome of CSE. Thus, for those patients with unfavorable outcome, we inquired if other risk factors had been encountered after discharge. However, there was no such case in this study cohort. The age of patients had been previously determined by other studies to be an important risk factor for mortality after SE [5, 24-26]. In this study, we did not find a significant relationship between age and functional outcome. This could be partially explained by the fact that there is no direct relationship between age and the severity of clinical conditions, and the latter has an important influence on patients' recovery. However, it should be noted that the age distribution in the sample group used in our study is different from previous studies. STESS which was created by Rossetti et al. was based on a SE database of 127 episodes occurring in 107 adult patients, in which more than $30 \%$ of the patients were $\geq 65$ years of age [24], whereas the median age of our sample group was 25.5 (IQR, 17-48) years of age, and only 11 of the $132(8.33 \%)$ patients were over 65 years of age. Given that older patients are less resistant to complications of SE and its treatment, such as pneumonia, this could result in a higher incidence of unfavorable outcomes in older patients. A study with a larger sample size and a stratification analysis is needed to clarify this.

Recent studies have indicated that encephalitis is an acute symptomatic etiology present in most cases of SE and is related to a poor prognostic outcome in developing countries [2, 13-17]. Patients with encephalitis were also determined in this study to constitute $35.6 \%$ of all cases of SE, $68.1 \%$ of whom had a score between 3 and 6 at three months post discharge, indicative of an unfavorable functional outcome, which is consistent with the results from previous studies. Therefore, encephalitis was chosen as an etiology variable in the prediction of the functional outcome for CSE patients, which was proven to be an independent predictor by multivariate logistic regression analysis.

NCSE in the END-IT scoring system refers to subtle SE. Subtle SE, a term used to describe SE with subtle clinical features of myoclonic jerks or nystagmus in association with EEG discharges, is a form of NCSE that develops from CSE if the latter has been treated insufficiently [27, 28]. A considerable percentage of

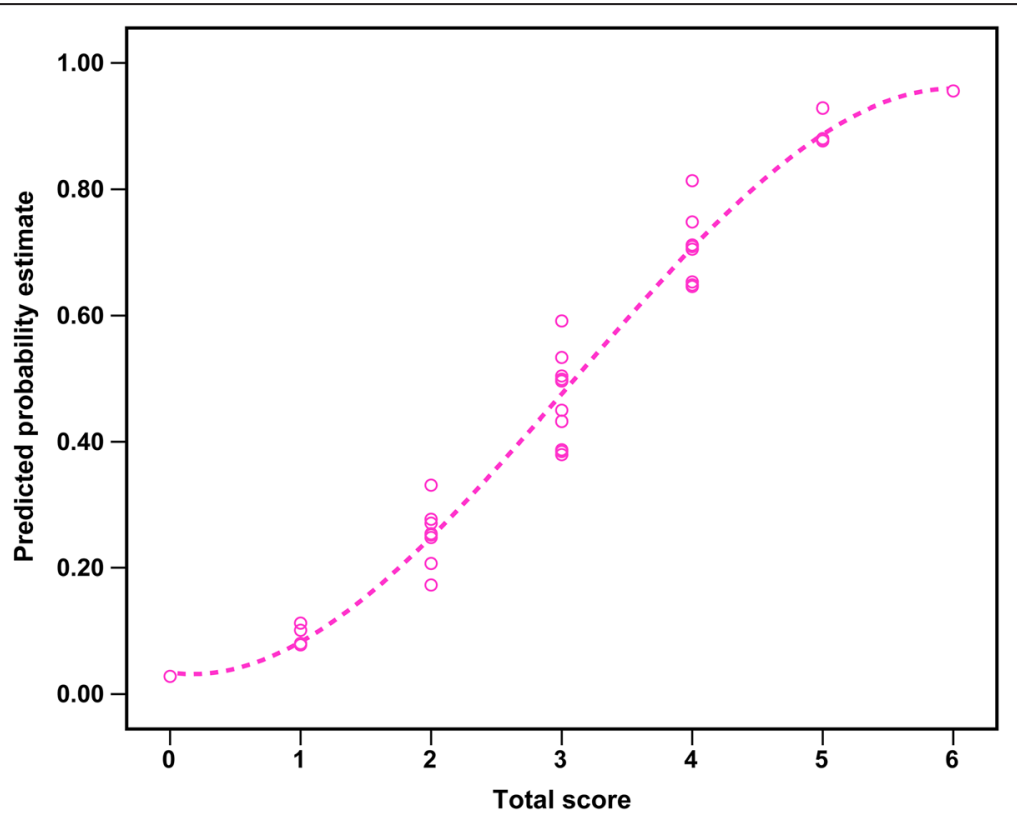

Fig. 2 END-IT scores and their corresponding predicted estimates for an unfavorable outcome 


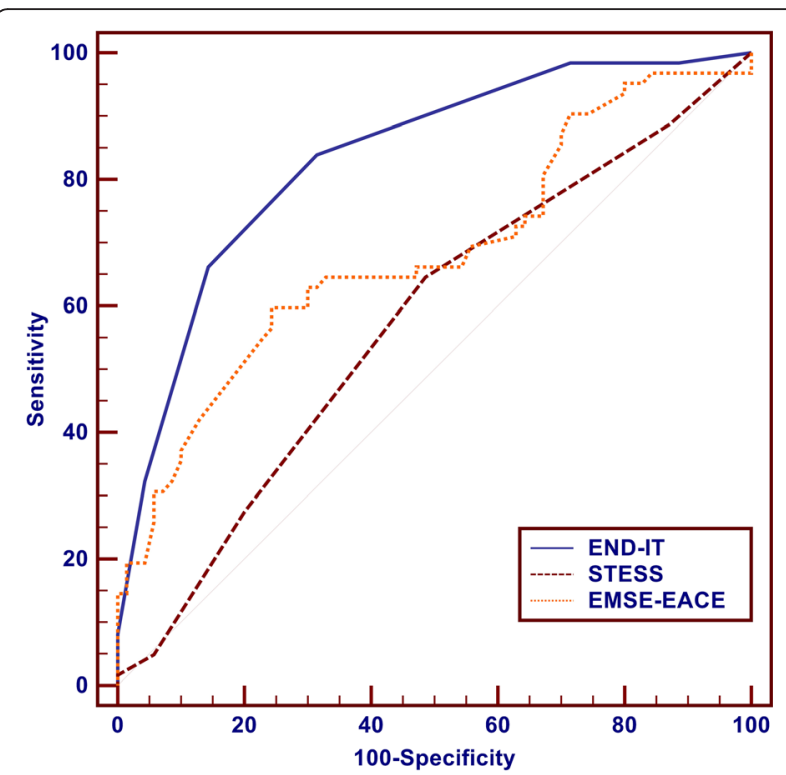

Fig. 3 Receiver operating characteristic curve (ROC) for END-IT, Status Epilepticus Severity Score (STESS) and Epidemiology based Mortality score in SE using etiology-age-comorbidity-EEG (EMSE-EACE). END-IT: AUC $=0.833,95 \% \mathrm{Cl}: 0.766-0.900 ;$ STESS: $A U C=0.573,95 \% \mathrm{Cl}:$ 0.478-0.668; EMSE-EACE: AUC $=0.683,95 \%$ Cl: 0.590-0.776

patients (47.7 \%) suffering from subtle SE, which might be due to structural or metabolic brain damage or an initial insufficient treatment, were found in our study. Previous studies have shown that the presence of NCSE, once CSE was under control, was associated with significant mortality and poor functional outcome $[29,30]$. Similarly, we also found that patients with NCSE had a higher proportion of unfavorable outcomes than those without NCSE.

Impairment of consciousness at the onset of SE can be indicative of extreme severity, and has also been shown to be independently related to death in previous retrospective studies [24, 30, 31]. In this study, we used a GCS score and a pupillary light reflex on N-ICU admission in order to evaluate the level of consciousness of each patient with SE. However, a significant relationship between these two variables and the functional outcome was not found. This may be due to the fact that patients on N-ICU admission have received an intravenous diazepam injection in the ambulance or emergency room, which could affect their level of consciousness.

Refractory SE is defined as the presence of SE either clinically or as determined by electrographic examination despite treatment with adequate doses of an initial benzodiazepine followed by a second acceptable AED $[10,32]$. The long-term outcome of 596 cases with refractory SE was analyzed by Ferlisi et al., the results of which indicated that $35 \%$ of the patients had died, $13 \%$ had a severe neurological deficit, and only $35 \%$ recovered to baseline [33]. Recent prospective studies have also shown that refractory SE appeared to be strongly associated with poor outcomes [34, 35]. Considering the controversy surrounding the definition of refractory SE, i.e., the number of AEDs that patients need to have failed and the duration of SE after the initiation of treatment [10], we chose several variables that reflect the features of refractory SE, including diazepam resistance, SE duration, use of three or more types of intravenous AEDs, a drug induced coma, and tracheal intubation, as candidate predictors when performing the statistical analysis. Although these variables were shown to be related to an unfavorable outcome in the univariate analysis, multivariate analysis demonstrated that only tracheal intubation functioned as an independent predictor. This could be explained by the fact that tracheal intubation was a consequence possibly linked to diazepam resistance, a longer SE duration, usage of three or more types of intravenous AEDs, and a drug induced coma. Meanwhile, tracheal intubation implied a high risk of respiratory failure, mechanical ventilation, pneumonia, and a longer ICU stay, which were the primary reasons for the poor outcome observed for refractory SE [36, 37].

Recently, Kilbride et al. investigated the clinical outcomes of 63 patients with prolonged refractory SE and found that a normal neuroimage at the onset of SE was associated with a favorable outcome [38]. In an effort to clarify the role of neuroimaging findings in the prediction

Table 5 Performance of the END-IT score

\begin{tabular}{|c|c|c|c|c|}
\hline End-it Score & Sensitivity (95 \% Cl) & Specificity (95 \% Cl) & PPV (95 \% Cl) & NPV $(95 \% \mathrm{Cl})$ \\
\hline 0 & $100.00(94.2-100.0)$ & $0.00(0.0-5.1)$ & $47.0(38.2-55.8)$ & \\
\hline 1 & $98.39(91.3-100.0)$ & $11.43(5.1-21.3)$ & $49.6(40.5-58.8)$ & 88.9 (48.9-99.8) \\
\hline 2 & $98.39(91.3-100.0)$ & $28.57(18.4-40.6)$ & $55.0(45.2-64.4)$ & $95.2(75.5-99.9)$ \\
\hline 3 & 83.87 (72.3-92.0) & $68.57(56.4-79.1)$ & $70.3(58.5-80.3)$ & $82.8(70.4-91.5)$ \\
\hline 4 & $66.13(53.0-77.7)$ & 85.71 (75.3-92.9) & $80.4(66.9-90.2)$ & $74.1(63.1-83.2)$ \\
\hline 5 & $32.26(20.9-45.3)$ & 95.71 (88.0-99.1) & $87.0(65.8-97.4)$ & $61.5(51.7-70.6)$ \\
\hline 6 & $8.06(2.7-17.8)$ & $100.00(94.9-100.0)$ & $100.0(47.8-100.0)$ & $55.1(46.0-63.9)$ \\
\hline
\end{tabular}

PV was study population specific

$P P V$ positive predictive value, NPV negative predictive value 


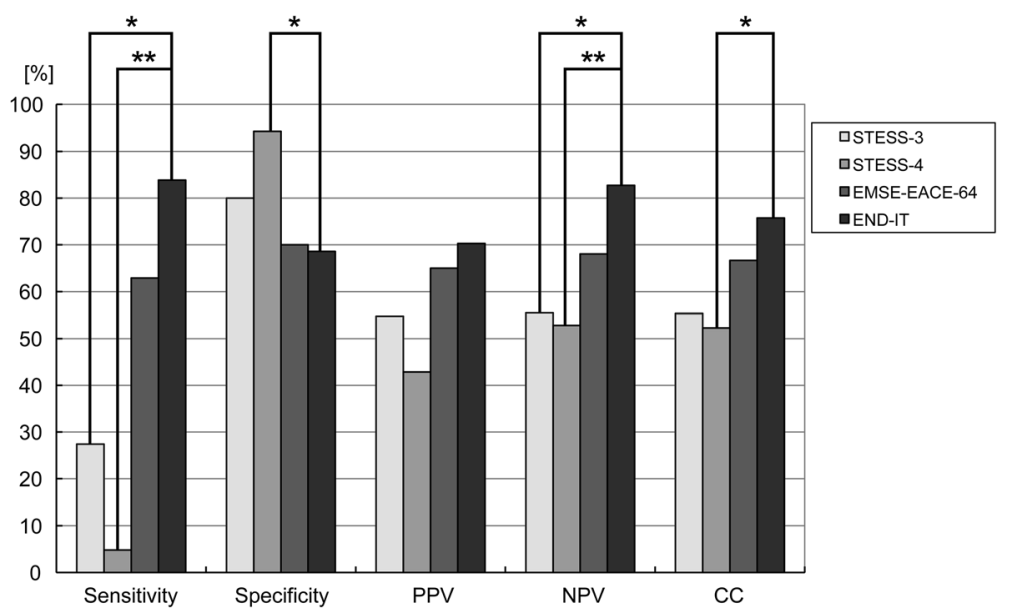

Fig. 4 Comparisons of sensitivity, specificity, positive predictive value (PPV) and negative predictive value (NPV), number of correctly classified (CC) patients between STESS-3, STESS-4, EMSE-EACE-64 and END-IT. Sensitivity: ${ }^{*} p=0.0000000028,{ }^{* *} p=0.0000000000000046$; specificity: ${ }^{*} p=$ 0.00012 ; NPV: ${ }^{*} p=0.0000030,{ }^{* *} p=0.000000099$; correctly classified: ${ }^{*} p=0.00037$. Level of significance corrected for multiple testing $p<0.00833$. STESS status epilepticus severity score, EMSE epidemiology based mortality score

of functional outcomes, taking into account the extent of brain damage evident on CT/MRI brain scans, we classified them into three types, in which unilateral responsible lesions and bilateral responsible lesions or the presence of diffuse cerebral edema were all significantly associated with unfavorable outcomes. In light of their contribution in multivariate models, unilateral lesions were assigned 1 point, whereas bilateral lesions or diffuse cerebral edema were each given 2 points in the END-IT scoring system. However, because many patients underwent only one imaging examination during N-ICU hospitalization, our study could not demonstrate if an evolution of imaging findings is predictive of a functional outcome.

The limitations of this study should be noted. First, the score was developed based upon data extracted from a relatively small sample size from a single university tertiary medical center. Thus, the results may not be able to be extrapolated to other settings or populations; hence, further validation in different settings is needed. Second, we collected the functional outcome data at three months after discharge, but not three months after onset of CSE. Since the functional outcome is time dependent, it could be influenced by some other factors, e.g., the discharge habits of the different involved physicians, and shortness of hospital beds for fast discharge. Third, our scoring system did not take into account serological variables, e.g., serum neuron-specific enolase (NSE). Although a few reports have previously shown that NSE may be a promising in vivo marker for brain injury after SE [39-42], its exact role in prognosis of CSE awaits further research. Fourth, we did not examine the role of certain EEG patterns in outcome prediction. Long-term EEG monitoring revealed that some patients often presented with several different kinds of EEG patterns, e.g., burst-suppression and postictal discharges could coexist with difference in sequence, which makes it difficult to interpret the relationship between a specific EEG pattern and a functional outcome. Lastly, we collected the follow-up information on function outcome of patients through telephone interviews but not clinical examination. This might result in some small biases in outcome information, since mRS based on telephone interview could be influenced by some complicating factors.

\section{Conclusions}

In this study, we developed a straightforward and practicable scoring system, termed END-IT, to predict the three-month functional outcome post discharge for CSE patients. The score comprises five in-hospital variables: encephalitis, NCSE, diazepam resistance, image, and tracheal intubation, and showed good predictive accuracy and discrimination power. However, it should be remembered that the END-IT score simply predicts the probability of functional outcome, with no judgment being made as to whether the patients continue to receive intensive treatment or not. Future studies are required for further validation and improvements upon this scoring system.

\section{Key messages}

- Forty-seven percent of patients with convulsive status epilepticus lasting more than 30 minutes suffered an unfavorable outcome.

- Encephalitis, NCSE, diazepam resistance, image abnormalities and tracheal intubation were independent predictors for unfavorable outcome. 
- Based on these five independent predictors and their coefficients in the logistic regression model, we established a 6-point scoring system, termed END-IT.

- The END-IT score showed good discriminative power as well as predictive accuracy.

\section{Additional file}

\section{Additional file 1: Baseline characteristics of 132 patients. (DOC $42 \mathrm{~kb}$ )}

\begin{abstract}
Abbreviations
AED: Antiepileptic drug; AUC: Area under the curve; CC: Correctly classified CCl: Charlson's Comorbidity Index; CSE: Convulsive status epilepticus; CT: Computed tomography; EACE: Etiology-age-comorbidity-EEG; EEG: electroencephalogram; EMSE: Epidemiology based mortality score; GCS: Glasgow Coma Scale; IQR: Interquartile range; IV: Intravenous; MRI: Magnetic resonance imaging; mRS: Modified Rankin Scale; NCSE: Nonconvulsive status epilepticus; N-ICU: Neurological intensive care unit; NPV: Negative predictive value; NSE: Neuron-specific enolase; PPV: Positive predictive value; ROC: Receiver operating characteristic curve; STESS: Status epilepticus severity score.
\end{abstract}

\section{Competing interests}

The authors declare that they have no competing interests.

\section{Authors' contributions}

Drs. QG, TpOY, and WJ designed the study. Drs. QG, TpOY, XIS, FY, CW, and TK collected data. Drs. QG, TpOY, XIS, FY, CW, TK, XGK and Jiang analyzed and interpreted the data. Drs. QG and XGK conducted the statistical analysis. Drs. QG and WJ drafted the manuscript. All authors critically revised the article and approved the final manuscript.

\section{Acknowledgements}

This work was supported by grants from the Natural Science Foundation of China (grant number 81571262) and from the Xijing Hospital Research Foundation (grant number XKZT09Z07, to W Jiang).

\section{Received: 2 December 2015 Accepted: 6 February 2016}

\section{Published online: 25 February 2016}

\section{References}

1. Betjemann JP, Lowenstein DH. Status epilepticus in adults. Lancet Neurol. 2015;14:615-24

2. Neligan A, Shorvon SD. Frequency and prognosis of convulsive status epilepticus of different causes: a systematic review. Arch Neurol. 2010;67:931-40.

3. Lowenstein DH, Alldredge BK. Status epilepticus at an urban public hospital in the 1980s. Neurology. 1993;43:483-8.

4. Aminoff MJ, Simon RP. Status epilepticus. Causes, clinical features and consequences in 98 patients. Am J Med. 1980;69:657-66.

5. Rossetti AO, Logroscino G, Milligan TA, Michaelides C, Ruffieux C, Bromfield EB. Status epilepticus severity score (STESS): A tool to orient early treatment strategy. J Neurol. 2008;255:1561-6.

6. Rossetti AO, Logroscino $G$, Bromfield EB. A clinical score for prognosis of status epilepticus in adults. Neurology. 2006;66:1736-8.

7. Leitinger M, Holler Y, Kalss G, Rohracher A, Novak HF, Hofler J, et al. Epidemiology-based mortality score in status epilepticus (EMSE). Neurocrit Care. 2015;22:273-82

8. Guidelines for epidemiologic studies on epilepsy. Commission on Epidemiology and Prognosis, International League Against Epilepsy. Epilepsia. 1993;34:592-6.

9. Towne AR, Pellock JM, Ko D, DeLorenzo RJ. Determinants of mortality in status epilepticus. Epilepsia. 1994:35:27-34.

10. Brophy GM, Bell R, Claassen J, Alldredge B, Bleck TP, Glauser T, et al. Guidelines for the evaluation and management of status epilepticus, Neurocrit Care. 2012;17:3-23.

11. Kalviainen R. Status epilepticus treatment guidelines. Epilepsia. 2007:48(8 Suppl):S99-S102
12. Beniczky S, Hirsch LJ, Kaplan PW, Pressler R, Bauer G, Aurlien H, et al. Unified EEG terminology and criteria for nonconvulsive status epilepticus. Epilepsia. 2013;54(6 Suppl):S28-9.

13. Chen L, Zhou B, Li JM, Zhu Y, Wang JH, Sander JW, et al. Clinical features of convulsive status epilepticus: a study of 220 cases in western China. Eur J Neurol. 2009;16:444-9.

14. Jayalakshmi S, Ruikar D, Vooturi S, Alladi S, Sahu S, Kaul S, et al. Determinants and predictors of outcome in super refractory status epilepticus-a developing country perspective. Epilepsy Res. 2014;108:1609-17.

15. Li Y, Tian L, Zeng T, Chen J, Chen L, Zhou D. Clinical features and outcome of super-refractory status epilepticus: a retrospective analysis in West China. Seizure. 2014;23:722-7.

16. Zhou B, Huang Y, Wang J, Zou Z, Chen L, Li J, et al. The aetiology of convulsive status epilepticus: a study of 258 cases in Western China. Seizure. 2014;23:717-21.

17. Kariuki SM, Kakooza-Mwesige A, Wagner RG, Chengo E, White S, Kamuyu G, et al. Prevalence and factors associated with convulsive status epilepticus in Africans with epilepsy. Neurology. 2015;84:1838-45.

18. Prasad A, Worrall BB, Bertram EH, Bleck TP. Propofol and midazolam in the treatment of refractory status epilepticus. Epilepsia. 2001;42:380-6.

19. Stecker MM, Kramer TH, Raps EC, O'Meeghan R, Dulaney E, Skaar DJ. Treatment of refractory status epilepticus with propofol: clinical and pharmacokinetic findings. Epilepsia. 1998;39:18-26.

20. Logroscino G, Hesdorffer DC, Cascino GD, Annegers JF, Bagiella E, Hauser WA. Long-term mortality after a first episode of status epilepticus. Neurology. 2002;58:537-41.

21. Charlson ME, Pompei P, Ales KL, MacKenzie CR. A new method of classifying prognostic comorbidity in longitudinal studies: development and validation J Chronic Dis. 1987;40:373-83.

22. van Swieten JC, Koudstaal PJ, Visser MC, Schouten HJ, van Gijn J. Interobserver agreement for the assessment of handicap in stroke patients. Stroke. 1988:19:604-7.

23. Bland JM, Altman DG. Multiple significance tests: the Bonferroni method. BMJ. 1995:310:170.

24. Rossetti AO, Hurwitz S, Logroscino G, Bromfield EB. Prognosis of status epilepticus: role of aetiology, age, and consciousness impairment at presentation. J Neurol Neurosurg Psychiatry. 2006;77:611-5.

25. Chin RF, Nevillea BG, Scotta RC. A systematic review of the epidemiology of status epilepticus. Eur J Neurol. 2004;11:800-10.

26. Koubeissi M, Alshekhlee A. In-hospital mortality of generalized convulsive status epilepticus: a large US sample. Neurology. 2007;69:886-93.

27. Maganti R, Gerber P, Drees C, Chung S. Nonconvulsive status epilepticus. Epilepsy Behav. 2008;12:572-86.

28. Meierkord $\mathrm{H}$, Holtkamp M. Non-convulsive status epilepticus in adults: clinical forms and treatment Lancet Neurol. 2007:6:329-39.

29. DeLorenzo RJ, Waterhouse EJ, Towne AR, Boggs JG, Ko D, DeLorenzo GA, et al. Persistent nonconvulsive status epilepticus after the control of convulsive status epilepticus. Epilepsia. 1998;39:833-40.

30. Sutter R, Kaplan PW, Ruegg S. Outcome predictors for status epilepticuswhat really counts. Nat Rev Neurol. 2013;9:525-34

31. Legriel S, Mourvillier B, Bele N, Amaro J, Fouet P, Manet $P$, et al. Outcomes in 140 critically ill patients with status epilepticus. Intensive Care Med. 2008; 34:476-80.

32. Rossetti AO, Lowenstein DH. Management of refractory status epilepticus in adults: still more questions than answers. Lancet Neurol. 2011;10:922-30.

33. Ferlisi M, Shorvon S. The outcome of therapies in refractory and superrefractory convulsive status epilepticus and recommendations for therapy. Brain. 2012;135:2314-28

34. Legriel S, Azoulay E, Resche-Rigon M, Lemiale V, Mourvillier B, Kouatchet A et al. Functional outcome after convulsive status epilepticus. Crit Care Med. 2010;38:2295-303.

35. Moghaddasi M, Joodat R, Ataei E. Evaluation of short-term mortality of status epilepticus and its risk factors. J Epilepsy Res. 2015;5:13-6.

36. Hocker S, Wijdicks EF, Rabinstein AA. Refractory status epilepticus: new insights in presentation, treatment, and outcome. Neurol Res. 2013;35:163-8.

37. Sutter R, Tschudin-Sutter S, Grize L, Fuhr P, Bonten MJ, Widmer AF, et al. Associations between infections and clinical outcome parameters in status epilepticus: a retrospective 5-year cohort study. Epilepsia, 2012:53:1489-97.

38. Kilbride RD, Reynolds AS, Szaflarski JP, Hirsch LJ. Clinical outcomes following prolonged refractory status epilepticus (PRSE). Neurocrit Care. 2013;18:374-85 
39. Correale J, Rabinowicz AL, Heck CN, Smith TD, Loskota WJ, DeGiorgio CM. Status epilepticus increases CSF levels of neuron-specific enolase and alters the blood-brain barrier. Neurology. 1998;50:1388-91.

40. DeGiorgio CM, Correale JD, Gott PS, Ginsburg DL, Bracht KA, Smith T, et al. Serum neuron-specific enolase in human status epilepticus. Neurology. 1995:45:1134-7.

41. DeGiorgio CM, Gott PS, Rabinowicz AL, Heck CN, Smith TD, Correale JD, Neuron-specific enolase, a marker of acute neuronal injury, is increased in complex partial status epilepticus. Epilepsia. 1996;37:606-9.

42. DeGiorgio CM, Heck CN, Rabinowicz AL, Gott PS, Smith T, Correale J. Serum neuron-specific enolase in the major subtypes of status epilepticus. Neurology. 1999;52:746-9.

Submit your next manuscript to BioMed Central and we will help you at every step:

- We accept pre-submission inquiries

- Our selector tool helps you to find the most relevant journal

- We provide round the clock customer support

- Convenient online submission

- Thorough peer review

- Inclusion in PubMed and all major indexing services

- Maximum visibility for your research

Submit your manuscript at www.biomedcentral.com/submit
() BioMed Central 